\title{
A cut short to death: Parl and Opa1 in the regulation of mitochondrial morphology and apoptosis
}

\author{
L Pellegrini ${ }^{\star, 1}$ and L Scorrano ${ }^{\star, 2}$
}

Mitochondria are crucial amplifiers of death signals. They release cytochrome $c$ and other pro-apoptotic factors required to fully activate effector caspases. This release is accompanied by fragmentation of the mitochondrial reticulum and by remodelling of the internal structure of the organelle. Here we review data supporting the existence of a regulatory network in the inner mitochondrial membrane that includes optic atrophy 1 (Opa1), a dynamin-related protein, and presenilin-associated rhomboidlike (Parl), a rhomboid protease. Opa1 regulates remodelling of the cristae independent of its effect on fusion. Cristae remodelling conversely requires Parl, which participates in the production of a soluble form of Opa1 retrieved together with the integral membrane one in oligomers that are disrupted early during apoptosis. Parl itself is regulated by proteolysis to generate a cleaved form, which in turn modulates the shape of the mitochondrial reticulum. Cleavage of Parl depends on its phosphorylation state around the cleavage site, implicating mitochondrial kinases and phosphatases in the regulation of mitochondrial shape.

Cell Death and Differentiation (2007) 14, 1275-1284; doi:10.1038/sj.cdd.4402145; published online 20 April 2007

Mitochondria not only represent the site of oxidative phosphorylation, which ensures most of the ATP required by the cell, but are also positioned at the centre of several signalling cascades. They uptake $\mathrm{Ca}^{2+}$ discharged from the endoplasmic reticulum (ER) to modulate the cytosolic transients of this second messenger; ${ }^{1}$ they participate in several anabolic and catabolic reactions involved in the buildup and disposal of essential cellular metabolites; ${ }^{2}$ as a by-product of their respiratory activity they produce reactive oxygen species (ROS) that can be used as second messengers by the cell; ${ }^{3}$ last, but not least, they are core apoptotic components that amplify the death signals coming from within the cell or from the outside. ${ }^{4}$

Mitochondrial involvement in apoptosis has been well characterized in the last 10 years. Its two main features include the release of proteins from the intermembrane space (IMS) and the initiation of a programme of dysfunction that includes the loss of the proton electrochemical gradient across the inner membrane (IM). Molecular mechanisms that are still not entirely clear and probably crosstalk mediate these two events. ${ }^{5}$ Nevertheless, the general consensus is that during apoptosis mitochondria release cytochrome $c$, the only soluble component of the respiratory chain, and other proteins such as second mitochondrial activator of caspases, endonuclease $G$ and apoptosis inducing factor (AIF) to activate fully the programme of cell degradation, in a manner controlled by proteins belonging to the $\mathrm{Bcl}-2$ family. ${ }^{6}$

While these changes in mitochondrial function and protein content are now recognized as core events during apoptosis, the notion that mitochondrial shape remained untouched during cell death stood unchallenged for many years. Classically, one of the major criteria to distinguish apoptosis from necrosis was the retrieval of morphological derangement of mitochondria in the latter but not in the former. In the recent years this assumption has been subverted by two observations: mitochondrial network undergoes fragmentation and topology of the IM is altered in concurrence with the functional and proteomic changes occurring to the apoptotic mitochondrion. $^{7}$ These observations prompted several groups to investigate the mechanisms regulating mitochondrial morphology in healthy and dying cells and a new, unexpected player came into the game: a rhomboid protease of the inner mitochondrial membrane (IMM) called presenilin-associated rhomboid-like (Parl), originally identified in a yeast two-hybrid screen as an interactor of presenilin, ${ }^{8}$ a protein involved in processing of the amyloid $\beta$-peptide. Here, we review the recent progresses in the regulation of mitochondrial shape

\footnotetext{
${ }^{1}$ Centre de Recherche Universite' Laval Robert Giffard, $2601 \mathrm{Ch}$. de la Canardiere, Quebec, Canada; ${ }^{2}$ Dulbecco-Telethon Institute, Venetian Institute of Molecular Medicine, Via Orus 2, Padova, Italy

*Corresponding authors: L Scorrano, Dulbecco-Telethon Institute, Venetian Institute of Molecular Medicine, Via Orus 2, Padova 35129, Italy. Tel: + 390497923221; Fax: + 390497923271; E-mail: luca.scorrano@ unipd.it or L Pellegrini, Centre de Recherche Université Laval Robert Giffard, 2601 Ch. de la Canardiere, Quebec, Canada. Tel: + 14186635000, Ext. 6879; Fax: + 14186638756; E-mail: Luca.Pellegrini@ crulrg.vlaval.ca

Keywords: apoptosis; rhomboids; mitochondria; cristae remodelling; Parl; morphology

Abbreviations: AIF, apoptosis inducing factor; Drp1, dynamin-related protein 1; ER, endoplasmic reticulum; I-Clips, intramembrane-cleaving proteases; IMM, inner mitochondrial membrane; IMS, intermembrane space; Mfn, mitofusin; Opa1, optic atrophy 1; Parl, presenilin-associated rhomboid-like; RIP, regulated intramembrane proteolysis; ROS, reactive oxygen species; TMH, transmembrane helix

Received 06.2.07; revised 05.3.07; accepted 09.3.07; Edited by P Nicotera; published online 20.4.07
} 
during cell life and death, focusing on the role of this mitochondrial rhomboid protease.

\section{Regulation of Mitochondrial Shape}

The functional versatility of mitochondria is paralleled by their morphological complexity. In certain cell types mitochondria are organized in networks of interconnected organelles. ${ }^{7}$ Ultrastructurally, the IM can be further subdivided in an inner boundary membrane and in the cristae compartment, bag-like folds of the IM connected to it via narrow tubular junctions. ${ }^{9}$ The ultrastructure and the reticular organization of the organelle is determined by mitochondria-shaping proteins that impinge on the equilibrium between fusion and fission processes.

Mitochondrial fission. In mammalian cells, mitochondrial division is regulated by dynamin related protein 1 (Drp1) and Fis1. ${ }^{10,11}$ The large GTPase Drp1 is a cytosolic dynaminrelated protein. Its inhibition or its downregulation result in a highly interconnected mitochondrial network. ${ }^{12}$ The same phenotype is caused by downregulation of hFis $1 .{ }^{11}$ Fis 1 is a $16 \mathrm{kDa}$ integral protein of the outer mitochondrial membrane, containing a single transmembrane (TM) domain and a tetratricopeptide repeat (TPR, involved in protein-protein interaction) domain facing the cytosol. ${ }^{13}$ In addition to these two players, endophilin B1, a member of the endophilin family of fatty acid acyl transferases that participate in endocytosis, has been shown to play a role in mitochondrial fission. ${ }^{14}$ Some evidence exists that Fis1 is the receptor on the outer membrane for Drp1, via its TPR. Drp1 is recruited to mitochondria and constriction of the membranes takes place by direct or indirect interaction with hFis $1 .{ }^{15}$ Levels of Fis1 and Drp1 and therefore mitochondrial fission are proteolytically controlled by the opposing action of sumoylation and ubiquitination. ${ }^{16-18}$ The role of endophilin B1 in this process is more elusive, but it might be similar to that of endophilin 1. During endocytosis, endophilin 1 builds complexes with dynamin I, the dynamin responsible for the severing of the neck of the nascent endocytic vesicle, and provides the required lipid modification. ${ }^{19}$

Mitochondrial fusion. Mitofusins (Mfn) 1 and 2 control mitochondrial fusion in mammals. They possess an $\mathrm{N}$ terminal GTPase domain, two TM domains spanning the outer mitochondrial membrane and two regions crucial for protein-protein interaction. ${ }^{20,21}$ Deletion of either Mfn impairs embryonic development in the mouse at different stages. ${ }^{22}$ The only dynamin-like GTPase so far identified in the IM is optic atrophy 1 (Opa1), mutated in dominant optic atrophy, the most common cause of inherited optic neuropathy. Opa1 promotes fusion, ${ }^{23,24}$ exists in eight different splice variants ${ }^{25}$ and its function is regulated by the mitochondrial rhomboid protease Parl, as we will see in detail in Cipolat et al. ${ }^{26}$ To promote fusion, Mfn1 docks two juxtaposed mitochondria via its second coiled coil domain. ${ }^{27}$ The role of the two Mfns in fusion seems to be different. Mfn1 has higher GTPase activity and induces fusion more efficiently than Mfn2. ${ }^{28}$ Furthermore, Opa1 requires Mfn1 to mediate fusion, while Mfn2 functions independently of Opa1. ${ }^{23}$ Mfn2 seems to have an additional role in determining the efficiency of mitochondrial oxidative metabolism. ${ }^{29}$ It is conceivable that relative expression levels of these players dictate mitochondrial morphology in different cell types and during development. Along this line, during differentiation from embryonic stem cells into cardiomyocytes, the remarkable changes in mitochondrial ultrastructure and reticular organization are accompanied by the reduction in the levels of Opa1 and by the upregulation of Mfn2. This is likely coordinated with the metabolic switch from glycolytic to oxidative $;^{30}$ indeed, the repression of Mfn2 in myofibres reduces myocyte respiration, further supporting the liaison between this protein and mitochondrial oxidative metabolism. ${ }^{29}$

\section{Function Follows Form: Consequences of Mitochondrial Shape Changes}

Changes in mitochondrial shape influence crucial cellular functions, from $\mathrm{Ca}^{2+}$ signalling ${ }^{31}$ to generation of $\mathrm{ROS},{ }^{32}$ to neuronal plasticity, ${ }^{33}$ to intermediate metabolism, ${ }^{29}$ even to lifespan. ${ }^{34}$ We will now outline the existing evidence pointing to a role for these proteins in several aspects of cell life and death.

Regulation of cellular morphology and movement. Mitochondria are not randomly positioned in the cytosol: they accumulate probably at sites of high-energy demand, or where they are required to locally regulate $\mathrm{Ca}^{2+}$ signalling. Mitochondrial movement is highly coordinated with changes in organelle morphology, required to produce mitochondria whose size is compatible with their transport by microtubule-associated cargoes. ${ }^{35}$ It is therefore expected that any perturbation of the fusion-fission equilibrium could eventually result in the subcellular distribution of mitochondria and therefore alter functions that critically depend on this. Expression of pro-fusion shaping proteins such as Opa1 and Mfn1 decreases the number of dendritic spines and synapses, showing a role for mitochondrial morphology in the determination of complex cellular patterns. ${ }^{33}$ One possibility is that perturbation of the fusion-fission equilibrium causes defects in neural morphogenesis as a consequence of perturbed mitochondrial transport. Ablation of Milton, a kinesin associated protein responsible for mitochondrial movement in Drosophila, results in the absence of mitochondria from synapses, which are otherwise normally formed. ${ }^{36,37}$ This would suggest that mitochondrial fusion-fission plays a specific role in morphogenesis, additional to its influence on movement of the organelle. Similarly, Campello et al. ${ }^{38}$ recently demonstrated that the shape of the mitochondrial reticulum regulates the ability of lymphocytes to migrate. Following chemotactic stimulation, mitochondria accumulate at the uropod of polarized lymphocytes. This depends on the activation of a fission programme and is a required step in the determination of a migrating phenotype. Moreover, induction of mitochondrial fission is per se able to induce a polarized appearance of these lymphocytes, further strengthening the role of mitochondrial fusion-fission in cellular morphogenesis and ultimately function. ${ }^{38}$ 
Production of ROS and lifespan. Mitochondria, together with peroxisomes, are the major source of ROS. This metabolic role assigns to these organelles a perhaps crucial function in the determination of lifespan (see Balaban et al. $^{39}$ for a review). Mitochondrial dynamics seems to play a role in production of ROS as well as in longevity. Accumulation of ROS in pathological conditions such as oxygen/glucose deprivation critically depends on Drp1-dependent fragmentation of the mitochondrial reticulum. ${ }^{32}$ The reduced ROS accumulation could explain the increased longevity observed in two Fungi lacking Drp1. ${ }^{34}$ How mitochondrial fission is required for ROS production and lifespan remains unclear, albeit a link between the two processes seems plausible. For example, ablation of p66shc also increases lifespan and reduces production of ROS from mitochondria. ${ }^{40}$ P66shc can function as an alternative electron acceptor for cytochrome $c$, eventually generating $\mathrm{H}_{2} \mathrm{O}_{2}$ as the final product of this redox reaction. ${ }^{41} \mathrm{~A}$ tempting, unifying hypothesis would imply that in fragmented mitochondria the pools of cytochrome $c$ and p66shc are in closer contact, causing an increase in the electron transfer from the former to the latter, and ultimately in the formation of ROS. According to this hypothesis, inhibition of mitochondrial fission would result in reduced p66shc-mediated $\mathrm{H}_{2} \mathrm{O}_{2}$ production.

$\mathrm{Ca}^{2+}$ signalling. Mitochondria actively participate in the regulation of $\mathrm{Ca}^{2+}$ signalling by taking up and releasing $\mathrm{Ca}^{2+}$ in response to physiological, inositol triphosphate coupled agonists. This process relies on the relative position of mitochondria in the cytosol, as well as on their juxtaposition to the ER, required for the production of microdomains of high $\left[\mathrm{Ca}^{2+}\right]$, essential for the activation of the low affinity mitochondrial $\mathrm{Ca}^{2+}$ uniporter. ${ }^{1}$ It is therefore conceivable that changes in mitochondrial shape influence mitochondrial participation in the $\mathrm{Ca}^{2+}$ game. This hypothesis is substantiated by the finding that excessive fission by Drp1 blocks propagation of $\mathrm{Ca}^{2+}$ waves, ${ }^{31}$ while Fis1 reduces refilling of $\mathrm{ER} \mathrm{Ca}^{2+}$ stores, probably by impairing capacitative $\mathrm{Ca}^{2+}$ entry from the plasma membrane. ${ }^{42}$ Surprisingly, Fis 1 promotes higher degrees of fragmentation than Drp1, yet its effect on the propagation of mitochondrial $\mathrm{Ca}^{2+}$ waves are apparently much lower. A possibility is that Drp1 has a specific but yet not characterized function on mitochondrial $\mathrm{Ca}^{2+}$ propagation, in addition to its effect on mitochondrial morphology.

There are indications that $\mathrm{Ca}^{2+}$ signalling can influence mitochondrial dynamics. Mitochondria move at resting $\left[\mathrm{Ca}^{2+}\right]_{\text {i }}$, while inositol 1,4,5-trisphosphate- or ryanodine receptor-mediated $\mathrm{Ca}^{2+}$ signals decrease mitochondrial motility. ${ }^{43}$ As movement is critically controlled by dynamics, $\mathrm{Ca}^{2+}$ is likely to coordinates both processes. Possible candidates for this integration are Miro-1 and Miro-2, two newly identified Rho-like GTPases homologues of yeast Gem1p. These proteins possess EF-hand domains, suggesting the ability to bind $\mathrm{Ca}^{2+} ; 4$ interact with the kinesin partner Milton to regulate mitochondrial movement; ${ }^{45}$ and once activated induce fragmentation and perinuclear aggregation of mitochondria. ${ }^{44}$
Apoptosis. Mitochondria amplify apoptotic signals by releasing cytochrome $c$ and other cofactors required to activate effector caspases. This release is regulated by the proteins of the $\mathrm{Bcl}-2$ family, which includes both anti- and pro-apoptotic members. The pro-apoptotic 'BH3-only' proteins 'sense' the death stimuli and transduce them to mitochondria, where they activate the multidomain proapoptotic proteins Bax and Bak, ultimately resulting in cytochrome $c$ release. Antiapoptotic members of the family act by sequestering $\mathrm{BH} 3-$ only proteins or in another model by keeping multidomains inactive. ${ }^{46,47}$

During apoptosis mitochondria remodel their inner structure to allow the bulk of cytochrome $c$ to be released from the cristae stores. ${ }^{48}$ The groups of Martinou and Youle demonstrated that mitochondria undergo massive and reversible fragmentation before the release of cytochrome $c{ }^{49,50} \operatorname{Drp} 1$ is involved in this fragmentation process and a dominantnegative mutant of Drp1 protects from release of cytochrome $c$ and death. ${ }^{49}$ Supporting this scenario, the partner of Drp1 on mitochondrial membranes, Fis1, is also a player in apoptosis: its overexpression leads to cytochrome $c$ release, while its ablation protects from cell death. ${ }^{11,51}$ Furthermore, fragmentation is the only known and essential involvement of mitochondria during developmental apoptosis of Caenorhabditis elegans. ${ }^{52}$ Not only the pro-fission proteins are activated during apoptosis, but Mfn1-dependent fusion is impaired, as elegantly shown by Karbowski, Youle, and colleagues. ${ }^{53}$ In principle, this could occur by blockage of the function of Mfn1 itself, or of its IM partner Opa1. ${ }^{23}$ This latter option is supported by the release of a fraction of Opa1 together with cytochrome $c$ early in the course of apoptosis. ${ }^{54}$ TIMMP8a is another protein of the IMS, which is released from mitochondria and has been reported to be involved in the translocation of Drp1 from the cytosol to the organelle, ${ }^{55}$ raising the question of whether Drp1 dependent fission occurs upstream or downstream of outer-membrane permeabilization. In sum, the release of pro-fusion proteins as well as of required cofactors for the activation of pro-fission ones could be a required step to ensure fragmentation of mitochondria during apoptosis. On the other hand, at this stage, we lack precise knowledge of whether fragmentation occurs invariably upstream of mitochondrial outer-membrane permeabilization. Further studies, using genetic models of Bax, Bak ablation as well as of inactivation of the apoptosome, are required to establish a causal and temporal sequence of mitochondrial permeabilization, effector caspases activation and mitochondrial fragmentation during apoptosis. The notion that mitochondrial fission is a step towards death is reinforced by the ability of overexpressed pro-fusion Mfn1 and Mfn2 to block apoptosis induced by stimuli that recruit the mitochondrial pathway, perhaps by interfering with Bax activation. ${ }^{56,57}$ An open question is why increased fission accelerates cell death. A unifying model proposed by Shore and co-workers ${ }^{58}$ implies cristae remodelling downstream of Drp1 activation, which in turn depends on mitochondrial $\mathrm{Ca}^{2+}$ uptake induced by the BH3-only molecule BIK. The action of Drp1 depends on another BH3-only pro-apoptotic acting as a second hit to permeabilize the outer membrane. On the other hand, under certain circumstances mitochondrial fission is not associated with death. For example, Bax, Bak doubly deficient cells 
undergo fragmentation following treatment with intrinsic stimuli, yet they are resistant to the induction of apoptosis. ${ }^{59}$ Moreover, a single conservative point mutation in the short IMS stretch of Fis1 dissociates its pro-fission from its prodeath activity. ${ }^{59}$ On the other extreme, fission by Drp1 can even protect from death induced by $\mathrm{Ca}^{2+}$-dependent apoptotic stimuli that require mitochondria to amplify deadly waves of this second messenger. ${ }^{31}$ In sum, while excessive mitochondrial fission appears to be almost invariantly associated with death, we cannot unequivocally conclude that it is required for the execution of mitochondrial apoptosis. Therefore, genetic models of ablation of pro-fission mitochondria-shaping proteins are needed to understand whether fragmentation is required for developmental as well as postdevelopmental apoptosis.

\section{Opa1 and the Cristae Remodelling Pathway in Apoptosis}

To ensure the complete release of cytochrome $c$, the ultrastructure of mitochondria changes in the early stages of apoptosis. A combination of electron tomography and physiological measurements identified a pathway of cristae remodelling characterized by the widening of the narrow tubular junction and by the fusion of individual cristae. These morphological changes support the mobilization of cytochrome $c$ from the cristae to the IMS, and eventually to the cytosol. ${ }^{48}$ Opa1, the first discovered mitochondria-shaping protein located at the level of the IMM, seems a natural candidate to regulate this pathway. Nevertheless, its role in mitochondrial fusion ${ }^{23}$ argue against an unique function in controlling the shape of the cristae and therefore their remodelling during cell death. As expected, downregulation of Opa1 leads to mitochondrial fragmentation. This is accompanied by organelle dysfunction and cytochrome $c$ release and interestingly by changes in mitochondrial ultrastructure. ${ }^{60}$ These results raised the possibility that Opa1 could as a matter of fact participate in the biogenesis of the cristae and regulate the cristae remodelling pathway.

Genetic dissection of the role of Opa1 in mitochondrial fusion and apoptosis. To address the relative role of Opa1 in fusion versus apoptosis we undertook a genetic analysis. The expression of Opa1 in cells deficient for Mfn 1 and Mfn 2, the other components of the fusion machinery, resulted equally efficient in protection from death induced by a variety of stimuli. Opa1 delayed cytochrome $c$ release also in the absence of its genetic partner in mitochondrial fusion Mfn1, thus dissecting its antiapoptotic role from its function in fusion of the mitochondrial membranes. High levels of active Opa1 prevented mobilization of cytochrome $c$ from the cristae, as a consequence of their stabilizing effect on the diameter of the tubular junction. This proceeds independently of Mfn1 and of mitochondrial fusion. A biochemical analysis revealed that Opa1 resides in several high-molecular weight (MW) complexes, suggesting the possibility of its oligomerization, a common property among dynamins and dynamin related proteins. ${ }^{61}$ Moreover, a detailed inspection of Opa1 localization in mitochondrial compartments revealed a minor fraction ( $\sim 5 \%$ of total Opa1) of the protein soluble in the IMS. Following an apoptotic stimulus Opa1 is found in lower MW complexes. This is mirrored by the disappearance of a chemically crosslinkable Opa1-containing complex, whose size is compatible with that of a trimer of Opa1. This oligomer contained both the integral IM, and the soluble IMS form of Opa1, as revealed by expression of differentially tagged Opa1s, targeted exclusively to the IM or the IMS. ${ }^{62} \mathrm{~A}$ model of Opa1 fate during apoptosis is presented in Figure 1.

These findings raised the question of how the small fraction of soluble Opa1 could be produced. Opa1 is a complex gene, which is alternatively spliced generating eight mRNA isoforms. One possibility was therefore that the soluble Opa1 corresponded to an isoform lacking the TM domain. On the other hand, it should be noted that the TM of Opa1 is encoded by exons 2 and 3, retrieved in all alternatively spliced isoforms of the protein. ${ }^{25}$ This suggested the possibility that Opa1, like its yeast orthologue Mgm1p, undergoes posttranscriptional modification leading to its proteolytic cleavage and to the production of this shorter IMS form by Rbd1p, a rhomboid protease ${ }^{63-65}$ whose orthologue in mammals is called Parl. $^{8}$

Rhomboids are a widely conserved polytopic-membraneprotein family. ${ }^{66}$ In $D$. melanogaster they activate the epidermal growth factor (EGF) signalling pathway, by proteolytically cleaving the EGF receptor ligands Spitz, Gurken and Keren. ${ }^{67}$ A serine protease catalytic dyad is conserved in all rhomboids, ${ }^{66}$ suggesting that they can cleave TM domains of integral membrane proteins. Therefore, they have been functionally assigned to a class of highly hydrophobic proteases involved in regulated intramembrane proteolysis (RIP). ${ }^{67}$

\section{The Rhomboids}

RIP is a new paradigm of signal transduction, which appears to be prominent in all forms of life. ${ }^{67}$ Under RIP, membranebound proteins undergo site-specific proteolysis within one of
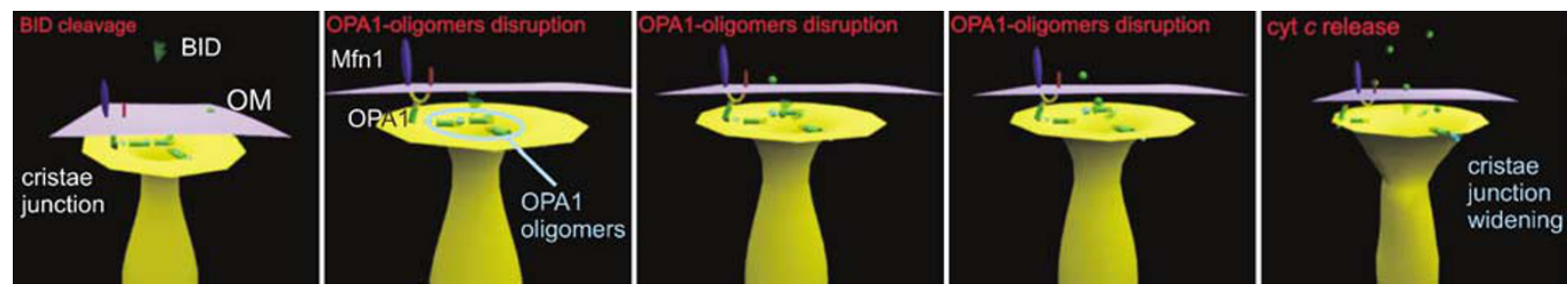

Figure 1 Fate of Opa1 oligomers during apoptosis. A sequence of cartoons depicting the changes occurring during cristae remodelling is presented. Note that for clarity, outer-membrane permeabilization required to ensure cytochrome $c$ release is not shown 
their transmembrane helix $(\mathrm{TMH})$. This processing releases a moiety of the protein that, through intracellular relocation or extracellular release, executes the signalling function of the membrane-tethered precursor protein. This mechanism influences processes as diverse as lipid metabolism, differentiation, response to unfolded proteins and mitochondrial morphology.

Enzymatically, RIP requires proteases that, despite the water-excluding environment of the lipid bilayer, are able to hydrolyze their TM substrates. This processing is executed by a very unique class of proteases, named intramembrane-cleaving proteases (I-Clips). ${ }^{68}$ To date, only three distinct I-Clip families have been discovered. The first is composed by a group of metalloproteases whose prototypic member is the human site-two protease that cleaves and activates sterol regulatory element binding proteins. ${ }^{69}$ The second family includes aspartic proteases, whose prototypic members are the presenilins (PSs) involved in cleavage of the amyloid $\beta$ protein precursor $(\mathrm{A} \beta \mathrm{PP})$ and Notch. ${ }^{70,71}$ This family also includes the signal peptide peptidase, which catalyses intramembrane proteolysis of signal sequence remnants and possibly also membrane proteins in the ER membrane. ${ }^{72}$ The third and most recently discovered I-Clip family are the rhomboids, ${ }^{67}$ which are the most evolutionary conserved one and, by implication, the first to emerge in life. ${ }^{66}$

In spite of the presence of rhomboids in the majority of modern life forms from all three primary superkingdoms, phylogenetic analysis suggests that this family has not been inherited from the last universal common ancestor. ${ }^{66}$ Instead, the tree topology indicates that this family emerged in certain bacterial lineages, had been widely disseminated by horizontal gene transfer (HGT) and then lost in some lineages. Archaea and eukaryotes have acquired rhomboids on several independent occasions. In particular, at least two HGT events have contributed to the origin of eukaryotic rhomboids, one of them yielding the $\mathrm{RHO}$ subfamily and the other one the Parl subfamily, with a possible additional HGT in plants, ${ }^{66}$ where rhomboids could regulate plastids morphology and activities.

The conserved core of rhomboid family members consists of six conserved $\mathrm{TMHs},{ }^{66}$ with the Ser and His residues required to form the catalytic dyad ${ }^{73}$ embedded in $\mathrm{TMH}-4$ and TMH-6, respectively. ${ }^{74}$ Consistent with the widely accepted hypothesis that I-Clips catalytic core is assembled within the plane of the membrane, recent crystallographic studies have shown that the catalytic dyad is found deep below the membrane surface. This indicates that the hydrolysis of the peptide bonds indeed takes place within the hydrophobic environment of the membrane bilayer. ${ }^{74}$

Eukaryotic members of the two subfamilies have different domain architecture organization. Proteins of the $\mathrm{RHO}$ subfamily, like the Drosophila developmental regulator rhomboid, ${ }^{75}$ typically have an extra $\mathrm{TMH} \mathrm{C}$ terminus to the $6-\mathrm{TMH}$ catalytic core, a ' $6+1$ ' structure. Members of the Parl subfamily, prototyped by Parl itself, have an extra TMH N terminus to the $6-\mathrm{TMH}$ catalytic core, a ' $1+6$ ' structure. $^{8}$ These additional TMHs imply the existence of a loop connecting them to the 6-TMH catalytic core. The functional role of this domain remains unknown.

\section{The Mitochondrial Rhomboids}

A yeast genetic screen aimed at identifying components involved in mitochondrial fusion led to the identification of two defective mutants, Ugo1 and Ugo2. ${ }^{76}$ Ugo2 was later described as a mutant of Pcp $1,{ }^{63}$ a gene encoding a rhomboid protease of the Parl subfamily.

Yeast cells lacking Pcp1p are defective in the processing of cytochrome $c$ peroxidase $1(\mathrm{Ccp} 1 \mathrm{p})^{65}$ and of Mgm1p, ${ }^{63-65}$ the orthologue of Opa1 and a key component of the mitochondrial fusion machinery. ${ }^{2}$ While the role of Ccp $1 p$ cleavage is still unknown, Mgm1p is a key player in the control of mitochondrial morphology. Consequently, the long tubular branched mitochondria of wild-type cells are replaced by partially fragmented ones in $\Delta \mathrm{Pcp} 1$ cells. $^{63,64}$ This phenotype is rescued by rhomboid-7 and Parl, the Drosophila and mammalian orthologues of Pcp1p. ${ }^{65}$ Thus, during evolution the ability of this rhomboid protease to cleave Mgm1p and therefore to regulate yeast mitochondrial morphology has been conserved. This is reflected by their localization in the $\mathrm{IM}^{77,78}$ and by their ' $1+6$ ' structure, with the catalytic serine and histidine located in $\mathrm{TMH} 5$ and $\mathrm{TMH} 7$, respectively. ${ }^{66}$

Despite of the functional and structural conservation of Pcp1p, rhomboid-7, and Parl, their N-terminal domain are unrelated. ${ }^{77}$ The $\mathrm{N}$-terminal region of Parl, which is in the matrix, ${ }^{78}$ spans the first 100 amino acids of the protein and shows no detectable similarity to any other available protein sequences. This domain of Parl, designated $\mathrm{P} \beta$ domain, is vertebrate-specific, as indicated by the notable conservation among mammals and, to a lesser extent, other vertebrates, but not between vertebrates and insects. ${ }^{77}$ The biological relevance of $\mathrm{P} \beta$ domain is evident from its sequence conservation. Indeed, in the four available mammalian Parl sequences, 58 of the 62 residues of the $\mathrm{P} \beta$ domain are invariant, and there are no insertions or deletions. This suggests that at least during mammalian evolution the $\mathrm{N}$ terminal region of Parl underwent strong purifying selection, which can be explained by functional constraints. In unconstrained sequences evolving neutrally, very few, if any, invariant residues would be expected to survive the $\sim 100$ million years of evolution separating mammalian orders. This analysis suggests that emergence of the $\mathrm{P} \beta$ domain at the outset of vertebrate evolution may be associated with the appearance of a new mechanism of regulation of Parl in mitochondria morphology and/or with the recruitment of Parl into novel mitochondrial pathways (Figure 2).

\section{Parl: A Shortcut to Regulate Apoptosis}

The ablation of Parl in the mouse does not affect development, yet has a major impact on postnatal growth and lifespan. Mice lacking Parl die between weeks 8 and 12 of cachexia sustained by multisystemic atrophy. The expected role of Parl in mitochondrial morphology as well as in oxidative phosphorylation could be ruled out in all the tissues analysed, where its ablation did not alter the morphology of the mitochondrial reticulum or mitochondrial respiration, irrespective of the substrate used by the organelle. Conversely, cells lacking Parl were more susceptible to apoptosis, as revealed both in situ and ex vivo, suggesting that a mechanism for the 
a

human
chimp
orangutan
dog
cow
mouse
human F

$\downarrow \alpha \quad \mid \beta$

\section{human} chimp orangutan dog mouse

$\mathrm{P} \quad \mathrm{PP}$

$\mid \beta$

$\alpha$

1 MAWRGWAORGWGCGOAWGASVGGRSCEELTAVLTPPOLLGRRFNFFIOOKCGFRKAPRKV 60

1 MAWRGWAQRGWGCGQAWGASVGGRSCEELTAVLTPPQLLGRRFNFFIQQKCGFRKAPRKV 60

1 MAWRGWAQRGWGCGQAWAASVGGRSCEELTAALTPPRLLGRRFNFFIQQKCGFRKAPRKV 60

1 MAWRGWAORGWGCGPAWAOPAGGRGCEELTAALAPPRLLGRRFDFFIOOKCGFRKAPRKV 60

1 MAWRGWAQRGWGCGQAWTLPVCGGSYEELTAALAPSRLLRRRFNFFIQQKCGFRKAPRKV 60

1 MALQGWVQRGWRCGPAWAPPLGG-GYRELSATQAP-RLLGRRFNLFVQQKCGFRKAPRKV 58

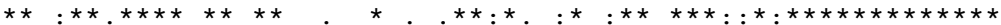

$\mathrm{TMH} 1$

EPRRSDPGTSGEAYKRSALIPPVEETVFYPSPYPIRSLIKPLFFTVGFTGCAFGSAAIWQ 120 EPRRSDTGTSGEAYKRSALI PPVEETVFYPSPYPIRSLIKPLFFTVGFTGCAFGSAAIWO 120 EPRRSDTGTSGEAYKRSALI PPVEETVFYPSPYPIRSLIKPLFFTVGFTGCAFGSAAIWQ 120 EPRRSDTGTSGEAYKRSALI PPVEETVFYPSPYPIRTLVKPLFFTVGFTGCAFGSAAIWQ 120 EPRRSD - -TSSEAYKRSALI PPVEETAFYPSPYPIRTLVKPLFFTVGFTGCAFGSAAIWQ 118 EPRRSDTGSSGEAYKRSALIPPLEETVFYPSPYPIRTLVKPFFFTIGFTGCAFGSAAIWQ 118

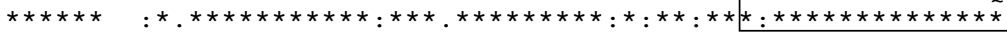

$\mathrm{TMH} 1$

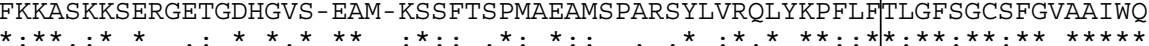

C human 1 MAWRGWAQRGWGCGQAWGASVGGRSCEELTAVLTPPQLLGRRFNFFIQQKC drosophila 1 MLMS----RAL-C-RSWLPQVARR-CHAN--VNVP--ILRINSGHPAARSC

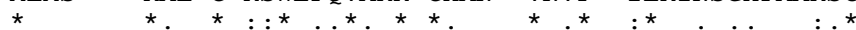
$\mid \alpha$ human GFRKAPRKVEPRRSDPGTSGEAYKRSALI PPVEETVFYPSPYPIRSLIKPLF TMH 1 drosophila RQIHSNRK-QSSNLKP-TTGE-------PAAAEQN- - - TPVPVN-- - - - TMH

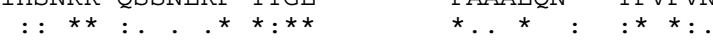

Figure 2 Evolutionary conservation of $\mathrm{N}$ terminus of Parl. (a) ClustalW alignments of the $\mathrm{N}$ terminus of mammalian orthologues of human $\mathrm{Parl}$ shows that the $\mathrm{P} \beta$ domain is strictly conserved in all species analyzed. (b) The $\mathrm{P} \beta$ domain is present and partially conserved in lower vertebrates, but not in invertebrates (c). Sites of $\alpha$ - and $\beta$-cleavage are shown. The amino acids whose phosphorylation $(\mathrm{P})$ status appears to control $\beta$-cleavage are indicated

massive atrophy observed. Parl was required to regulate the kinetics of cytochrome $c$ release from mitochondria. More specifically, it did not influence the actual efflux of this molecule from the organelle, but influenced the pathway of cristae remodelling controlled by Opa1. This function required the integrity of the catalytic dyad of Parl, as substantiated by the inability of a dyad mutant to complement Parl-/- cells. A genetic analysis revealed that Opa1 and Parl are part of the same pathway and that Parl is positioned upstream of Opa1 in the control of apoptosis. This raised the possibility that Parl was involved in the regulation of Opa1 oligomerization (Figure 3). In Parl-/- mitochondria, Opa1-containing oligomers were reduced and apoptotic stimulation disrupted them much faster. Moreover, a detailed biochemical analysis showed that levels of IMS Opa1 were reduced by approximately $50 \%$ in Parl-/- mitochondria. ${ }^{26}$ This partial reduction could explain why in the absence of Parl cristae are not basally deranged, yet undergo increased remodelling during apoptosis. The retrieval of a substantial fraction of soluble Opa1 in Parl-/mitochondria implies that Parl is probably not the only protease involved in Opa1 processing. For example, it has been reported that Paraplegin, an IMM mAAA metalloprotease, participates in the cleavage of Opa1 to produce a short isoform that is fusion incompetent. ${ }^{79,80}$ It should be noted that Paraplegin faces the matrix and that the mitochondrial processing peptidase already trims most of the matrix residues of Opa1. ${ }^{81}$ At this stage a plausible scenario deserving further investigation is that Paraplegin and Parl somehow cooperate in the cleavage of Opa1. For example, Paraplegin (or one of the other mAAA proteases that form the

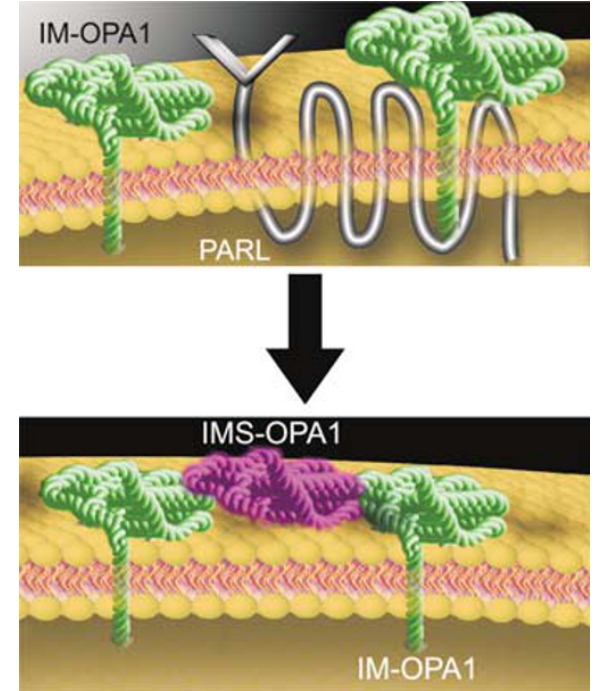

Figure 3 Parl and Opa1 interaction in the production of soluble Opa1. IMOPA1: IMM integral Opa1; IMS-OPA1, soluble, IMS Opa1

mAAA complex with paraplegin ${ }^{82}$ ) could participate in the activation of Parl at the level of its $\mathrm{N}$-terminal $\mathrm{P} \beta$ domain. This would imply the existence of a network of regulation that extends from protein precursor processing to mitochondrial morphology, to apoptosis. This network could be crucial to in the 'quality control' of mitochondria, coding damages of different intensity into an array of different responses that can vary from autophagy to cell death. 


\section{Regulating the Regulator}

The matrix $\mathrm{N}$-terminal $\mathrm{P} \beta$ domain of $\mathrm{Parl}$ undergoes two consecutive cleavage events, termed $\alpha$ and $\beta .{ }^{77}$ The proximal $\alpha$-cleavage $\left({ }_{52}\right.$ GlyPhe $\left._{53}\right)$ is a constitutive processing associated with the protein import in the mitochondria. ${ }^{77}$ Therefore, it is probably executed by one of the two proteases that typically are responsible for the import of most IMM proteins, the mitochondrial processing peptidase (MPP) and the innermembrane peptidase (IMP). ${ }^{83}$

The distal $\beta$-cleavage $\left({ }_{77} \operatorname{Ser} \downarrow \mathrm{Ala}_{78}\right.$ ) is regulated through a mechanism of proteolysis requiring Parl activity supplied in trans: a catalytically inactive Parl is not subjected to $\beta$-cleavage unless wild-type Parl was co-expressed. ${ }^{77}$ The identity of the matrix protease that cleaves Parl at the $\beta$ cleavage site remains elusive. However, Parl $\beta$-cleavage is unlikely to be executed by Parl itself since the cleavage site is not embedded within a hydrophobic helix ( ${ }_{53}$ FRKAPRKVEPRRSDPGTSGEAYKRSALIPPVEETVFYPSPYPIR-

SLIK $_{100}$ ), a strict requirement for rhomboids-mediated intramembrane proteolysis. ${ }^{74} \beta$-cleavage appears to require strict sequence conservation, as it is blocked when any of the four residues surrounding the site of cleavage are changed in Glu residues $\left({ }_{76} R S \downarrow A L_{79}\right) .{ }^{77}$ Since proteases implicated in the import of mitochondrial proteins do not have sequence specificity requirements, ${ }^{83} \beta$-cleavage is thus unlikely mediated by a protease of the import machinery, such as MPP and IMP. As mentioned above, mAAA proteases could supply the proteolytic activity required for $\beta$-cleavage of Parl.

$\beta$-Cleavage confers a gain of function in Parl-mediated regulation of mitochondria morphology. Mitochondrial fragmentation by overexpressed Parl was abolished when $\beta$ cleavage was abolished by removing or mutating the cleavage site. ${ }^{78}$ The functional importance of $\beta$-cleavage in the regulation of mitochondrial dynamics is further supported by the fact that a sophisticated mechanism of regulation of this processing exists in vivo. Endogenous and transfected Parl is constitutively and completely phosphorylated at three residues placed in close proximity to the $\beta$-cleavage site Ser $_{65}$, $\mathrm{Thr}_{69}$, Ser $\left._{70}\right)$. Since hyperphosphorylation of Parl blocks $\beta$ cleavage ${ }^{78}$ dephosphorylation appears to be required to allow this processing, thereby implicating a matrix phosphatase in the regulation of mitochondrial morphology (Figure 4). The identification of Parl kinase and phosphatase will therefore disclose the pathways implicated in sensing and transducing signals that impinge on mitochondrial shape. The discovery that phosphorylation and cleavage of Parl impacts on mitochondrial dynamics provides a novel rationale to interpret the contrasting reports on the cell and tissue specific effects of mitochondria-shaping proteins. ${ }^{23,84}$

The identification of the Parl kinase/phosphatase couple is even more important given our limited knowledge of reversible phosphorylation in mitochondria. To date, the only known example of this process within the mitochondrial IMS or matrix compartment are limited to the E1 subunits of the pyruvate and branched-chain $\alpha$-ketoacid dehydrogenase complexes. $^{85,86}$ The handful of kinases shown to reside inside the organelle include branched-chain $\alpha$-ketoacid dehydrogenase kinase, pyruvate dehydrogenase kinase, PKA, few isoforms of protein kinase $\mathrm{C}(\mathrm{PKC}), \mathrm{GSK} 3 \beta, \mathrm{CK} 2$ and PINK1. ${ }^{87}$ Their importance is substantiated by the finding that they can be mutated in major diseases, such as Parkinson's ${ }^{88}$ and diabetes. ${ }^{89}$ This small number of mitochondrial kinases (and phosphatases) is likely to increase in the near future especially taking into consideration the great number of mitochondrial functions (such as ATP synthesis, mtDNA replication, ROS production and scavenging, cristae remodelling and cytochrome $c$ release during apoptosis) that can be regulated through phosphorylation switches.

\section{Retrograde Regulation: an Additional Function for Parl?}

Retrograde regulation is a recently emerged field of mitochondrial research that involves the role of this organelle in intracellular signalling. ${ }^{90}$ Implicit in this definition is that mitochondrial signalling is opposite in direction to that of the more familiar anterograde regulation characterized by the transfer of information and material from the nucleus and cytoplasm to mitochondria.

It is now well established that cells modulate nuclear gene expression in response to mitochondrial activities and dysfunctions. For instance, impaired mitochondrial oxidative phosphorylation or mitochondrial DNA loss activates the Rtg1/ Rtg3 transcription factor complex, triggering anaplerotic reactions to maintain cellular glutamate levels in the absence of full TCA cycle activity. ${ }^{90}$ Inhibition of electron transport by antimycin $A$ as well as dissipation of the mitochondrial

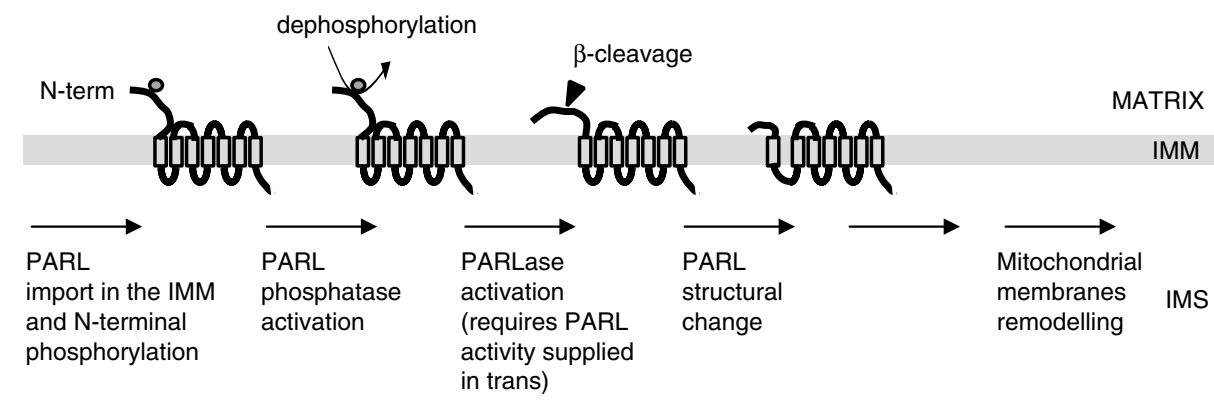

Figure 4 Cartoon of Parl dephosphorylation, cleavage and activation. Steps towards Parl activation are presented 
membrane potential by CCCP were also found to result in the repression of genes. ${ }^{91}$ Thus, the definition of retrograde regulation includes nuclear responses to changes in the functional state of mitochondria.

How do mitochondria control nuclear activities? Pioneering studies in the yeast Saccharomyces cerevisiae have shown that peptides extruded from the mitochondria participate in the regulation of nuclear genes. ${ }^{92}$ The first evidence for a release of peptides from mitochondria came from the observation that peptides derived from mitochondrially encoded proteins were detected at the cell surface of mammalian cells in association with major histocompatibility antigen class I molecules. ${ }^{93}$ Today, over 270 peptides, ranging in size between 6 and 26 amino acids, have been identified to be continuously exported from the matrix to the cytosol in an ATP- and temperaturedependent manner. ${ }^{94}$ They result from the catabolism of over 40 matrix and IMM proteins. Deletion of the yeast YME1 gene, which encodes the IM i-AAA protease, abolishes a large number of these peptides and leads to the induction of nuclear genes that regulate mitochondrial gene expression and biogenesis of the respiratory chain. ${ }^{95}$ This suggests that peptides released from the mitochondria execute a mitochondria-to-nucleus signalling pathway which allows the readjustment of the biogenesis of the respiratory chain in response to altered activity of the $F_{1} F_{0}$-ATP synthase.

The 25-amino acid-long peptide released in the matrix by Parl $\beta$-cleavage, termed $\mathrm{P} \beta$ peptide, can be efficiently targeted to the nucleus when transfected. ${ }^{77}$ In addition, the $\mathrm{P} \beta$ peptide can be found in the nucleus, suggesting a role for it in mitochondria-to-nucleus signalling. Although further studies are required to confirm this possibility, participation of the $\mathrm{P} \beta$ peptide in retrograde regulation would bring Parl within the mainstream concept of RIP, whereby a signalling protein is subjected to highly regulated release. Unlike other I-CliPs, however, the case of Parl would be the first example of RIP where the putative signalling moiety is part of the I-CliP itself.

\section{Conclusions}

Mitochondria participated in the most diverse cellular reactions, yet our knowledge of how these are regulated is extremely scarce. The individuation of a regulatory network in the IMM comprised of Parl, Opa1mAAA proteases and a yet unidentified kinase/phosphatase couple, with implications in mitochondrial morphology, metabolism, apoptosis and perhaps even gene expression opens new, unexpected avenues to investigate the role of this organelle in life and death of the cell.

Acknowledgements. LP is a FRSQ Scholar. Research in the Pellegrini laboratory is supported by grants from NSERC, CIHR, CFI, NCIC and the CRULRG. LS is an Assistant Telethon Scientist of the Dulbecco-Telethon Institute and an EMBO Young Investigator. Research in his lab is supported by Telethon Italy, AIRC Italy, UMDF USA, MDA USA, Fondazione Mariani, HFSPO, EMBO.

1. Rizzuto R, Bernardi P, Pozzan T. Mitochondria as all-round players of the calcium game. J Physiol 2000; 529 (Part 1): 37-47.

2. Dimmer KS, Scorrano L. De)constructing mitochondria: what for? Physiology (Bethesda) 2006; 21: 233-241.
3. Rhee SG. CELL SIGNALING: H2O2, a Necessary Evil for Cell Signaling. Science 2006; 312: $1882-1883$.

4. Green DR, Kroemer G. The pathophysiology of mitochondrial cell death. Science 2004 305: 626-629.

5. Bernardi $\mathrm{P}$, Petronilli V, Di Lisa F, Forte M. A mitochondrial perspective on cell death. Trends Biochem Sci 2001; 26: 112-117.

6. Scorrano L, Korsmeyer SJ. Mechanisms of cytochrome c release by proapoptotic BCL-2 family members. Biochemical Biophysical Res Comm 2003; 304: 437-444.

7. Cereghetti GM, Scorrano L. The many shapes of mitochondrial death. Oncogene 2006; 25 4717-4724.

8. Pellegrini L, Passer BJ, Canelles M, Lefterov I, Ganjei JK, Fowlkes BJ et al. PAMP and PARL, two novel putative metalloproteases interacting with the $\mathrm{COOH}$-terminus of Presenilin-1 and -2. J Alzheimers Dis 2001; 3: 181-190.

9. Frey TG, Mannella CA. The internal structure of mitochondria. Trends Biochem Sci 2000; 25: 319-324.

10. Smirnova E, Griparic L, Shurland DL, van der Bliek AM. Dynamin-related protein Drp1 is required for mitochondrial division in mammalian cells. Mol Biol Cell 2001; 12 2245-2256.

11. James DI, Parone PA, Mattenberger $\mathrm{Y}$, Martinou JC. hFis1, a novel component of the mammalian mitochondrial fission machinery. J Biol Chem 2003; 278: 36373-36379.

12. Smirnova E, Shurland D-L, Ryazantsev SN, van der Bliek AM. A human dynamin-related protein controls the distribution of mitochondria. J Cell Biol 1998; 143: 351-358.

13. Mozdy AD, McCaffery JM, Shaw JM. Dnm1p GTPase-mediated mitochondrial fission is a multi-step process requiring the novel integral membrane component Fis $1 \mathrm{p}$. J Cell Biol 2000; 151: 367-380.

14. Karbowski M, Jeong SY, Youle RJ. Endophilin B1 is required for the maintenance of mitochondrial morphology. J Cell Biol 2004; 166: 1027-1039.

15. Yoon $\mathrm{Y}$, Krueger EW, Oswald BJ, McNiven MA. The mitochondrial protein $\mathrm{hFis} 1$ regulates mitochondrial fission in mammalian cells through an interaction with the dynamin-like protein DLP1. Mol Cell Biol 2003; 23: 5409-5420.

16. Harder $Z$, Zunino R, McBride $H$. Sumo1 conjugates mitochondrial substrates and participates in mitochondrial fission. Curr Biol 2004; 14: 340-345.

17. Nakamura N, Kimura Y, Tokuda M, Honda S, Hirose S. MARCH-V is a novel mitofusin 2 and Drp1-binding protein able to change mitochondrial morphology. EMBO Rep 2006; 7: 1019-1022.

18. Yonashiro R, Ishido S, Kyo S, Fukuda T, Goto E, Matsuki $Y$ et al. A novel mitochondrial ubiquitin ligase plays a critical role in mitochondrial dynamics. EMBO J 2006; 25 3618-3626.

19. Schmidt A, Wolde M, Thiele C, Fest W, Kratzin H, Podtelejnikov AV et al. Endophilin I mediates synaptic vesicle formation by transfer of arachidonate to lysophosphatidic acid. Nature 1999; 401: 133-141.

20. Legros F, Lombes A, Frachon P, Rojo M. Mitochondrial fusion in human cells is efficient, requires the inner membrane potential, and is mediated by mitofusins. Mol Biol Cell 2002 13: 4343-4354

21. Santel A, Fuller MT. Control of mitochondrial morphology by a human mitofusin. J Cell Sci 2001; 114 (Part 5): 867-874.

22. Chen H, Detmer SA, Ewald AJ, Griffin EE, Fraser SE, Chan DC. Mitofusins Mfn1 and Mfn2 coordinately regulate mitochondrial fusion and are essential for embryonic development. J Cell Biol 2003; 160: 189-200.

23. Cipolat S, de Brito OM, Dal Zilio B, Scorrano L. OPA1 requires mitofusin 1 to promote mitochondrial fusion. Proc Natl Acad Sci USA 2004; 101: 15927-15932.

24. Meeusen S, DeVay R, Block J, Cassidy-Stone A, Wayson S, McCaffery JM et al. Mitochondrial inner-membrane fusion and crista maintenance requires the dynamin-related GTPase Mgm1. Cell 2006; 127: 383-395.

25. Delettre C, Griffoin JM, Kaplan J, Dollfus H, Lorenz B, Faivre L et al. Mutation spectrum and splicing variants in the OPA1 gene. Hum Genet 2001; 109: 584-591.

26. Cipolat S, Rudka T, Hartmann D, Costa V, Serneels L, Craessaerts K et al. Mitochondria rhomboid PARL regulates cytochrome $c$ release during apoptosis via OPA1-dependent cristae remodeling. Cell 2006; 126: 163-175.

27. Koshiba T, Detmer SA, Kaiser JT, Chen H, McCaffery JM, Chan DC. Structural basis of mitochondrial tethering by mitofusin complexes. Science 2004; 305: 858-862.

28. Ishihara N, Eura $\mathrm{Y}$, Mihara $\mathrm{K}$. Mitofusin 1 and 2 play distinct roles in mitochondrial fusion reactions via GTPase activity. J Cell Sci 2004; 117 (Part 26): 6535-6546.

29. Bach D, Pich S, Soriano FX, Vega N, Baumgartner B, Oriola J et al. Mitofusin-2 determines mitochondrial network architecture and mitochondrial metabolism. A novel regulatory mechanism altered in obesity. J Biol Chem 2003; 278: 17190-17197.

30. Chung S, Dzeja PP, Faustino RS, Perez-Terzic C, Behfar A, Terzic A. Mitochondrial oxidative metabolism is required for the cardiac differentiation of stem cells. Nat Clin Pract Cardiovasc Med 2007; 4 (Suppl 1(S1)): S60-S67.

31. Szabadkai G, Simoni AM, Chami M, Wieckowski MR, Youle RJ, Rizzuto R. Drp-1dependent division of the mitochondrial network blocks intraorganellar $\mathrm{Ca}^{2+}$ waves and protects against $\mathrm{Ca}^{2+}$-mediated apoptosis. Mol Cell 2004; 16: 59-68.

32. Yu T, Robotham JL, Yoon Y. Increased production of reactive oxygen species in hyperglycemic conditions requires dynamic change of mitochondrial morphology. Proc Nat Acad Sci USA 2006; 103: 2653-2658.

33. Li Z, Okamoto K, Hayashi Y, Sheng M. The importance of dendritic mitochondria in the morphogenesis and plasticity of spines and synapses. Cell 2004; 119: 873-887. 
34. Scheckhuber CQ, Erjavec N, Tinazli A, Hamann A, Nystrom T, Osiewacz HD. Reducing mitochondrial fission results in increased life span and fitness of two fungal ageing models. Nat Cell Biol 2007; 9: 99-105.

35. De Vos KJ, Allan VJ, Grierson AJ, Sheetz MP. Mitochondrial function and actin regulate dynamin-related protein 1-dependent mitochondrial fission. Curr Biol 2005; 15: 678-683.

36. Stowers RS, Megeath LJ, Gorska-Andrzejak J, Meinertzhagen IA, Schwarz TL. Axona transport of mitochondria to synapses depends on milton, a novel Drosophila protein Neuron 2002; 36: 1063-1077.

37. Gorska-Andrzejak J, Stowers RS, Borycz J, Kostyleva R, Schwarz TL, Meinertzhagen IA Mitochondria are redistributed in Drosophila photoreceptors lacking milton, a kinesinassociated protein. J Comp Neurol 2003; 463: 372-388.

38. Campello S, Lacalle RA, Bettella M, Manes S, Scorrano L, Viola A. Orchestration of lymphocyte chemotaxis by mitochondrial dynamics. J Exp Med 2006; 203 2879-2886.

39. Balaban RS, Nemoto S, Finkel T. Mitochondria, oxidants, and aging. Cell 2005; 120 483-495.

40. Migliaccio E, Giorgio M, Mele S, Pelicci G, Reboldi P, Pandolfi PP et al. The p66shc adaptor protein controls oxidative stress response and life span in mammals. Nature 1999; 402: 309-313.

41. Giorgio M, Migliaccio E, Orsini F, Paolucci D, Moroni M, Contursi C et al. Electron transfer between cytochrome $\mathrm{C}$ and $\mathrm{p66Shc}$ generates reactive oxygen species that trigge mitochondrial apoptosis. Cell 2005; 122: 221-233.

42. Frieden M, James D, Castelbou C, Danckaert A, Martinou JC, Demaurex N. Ca(2+) homeostasis during mitochondrial fragmentation and perinuclear clustering induced by hFis1. J Biol Chem 2004; 279: 22704-22714.

43. Yi M, Weaver D, Hajnoczky G. Control of mitochondrial motility and distribution by the calcium signal: a homeostatic circuit. J Cell Biol 2004; 167: 661-672.

44. Frederick RL, McCaffery JM, Cunningham KW, Okamoto K, Shaw JM. Yeast Miro GTPase Gem1p, regulates mitochondrial morphology via a novel pathway. J Cell Biol 2004; 167 87-98.

45. Glater EE, Megeath LJ, Stowers RS, Schwarz TL. Axonal transport of mitochondria requires milton to recruit kinesin heavy chain and is light chain independent. J Cell Biol 2006; 173: 545-557.

46. Danial NN, Korsmeyer SJ. Cell death: critical control points. Cell 2004; 116: 205-219.

47. Puthalakath $\mathrm{H}$, Strasser $\mathrm{A}$. Keeping killers on a tight leash: transcriptional and posttranslational control of the pro-apoptotic activity of BH3-only proteins. Cell Death Differ 2002; 9: 505-512

48. Scorrano L, Ashiya M, Buttle K, Weiler S, Oakes SA, Mannella CA et al. A distinct pathway remodels mitochondrial cristae and mobilizes cytochrome $c$ during apoptosis. Dev Cell 2002; 2: 55-67.

49. Frank S, Gaume B, Bergmann-Leitner ES, Leitner WW, Robert EG, Catez F et al. The role of dynamin-related protein 1, a mediator of mitochondrial fission, in apoptosis. Dev Cell 2001; 1: 515-525.

50. Martinou I, Desagher S, Eskes R, Antonsson B, Andre E, Fakan S et al. The release of cytochrome $c$ from mitochondria during apoptosis of NGF-deprived sympathetic neurons is a reversible event. J Cell Biol 1999; 144: 883-889.

51. Lee YJ, Jeong SY, Karbowski M, Smith CL, Youle RJ. Roles of the mammalian mitochondrial fission and fusion mediators Fis1, Drp1, and Opa1 in apoptosis. Mol Biol Cell 2004; 15: 5001-5011.

52. Jagasia R, Grote P, Westermann B, Conradt B. DRP-1-mediated mitochondria fragmentation during EGL-1-induced cell death in C. elegans. Nature 2005; 433 754-760.

53. Karbowski M, Arnoult D, Chen H, Chan DC, Smith CL, Youle RJ. Quantitation of mitochondrial dynamics by photolabeling of individual organelles shows that mitochondrial fusion is blocked during the Bax activation phase of apoptosis. J Cell Biol 2004; 164 : 493-499.

54. Arnoult D, Grodet A, Lee YJ, Estaquier J, Blackstone C. Release of OPA1 during apoptosis participates in the rapid and complete release of cytochrome $c$ and subsequent mitochondrial fragmentation. J Biol Chem 2005; 280: 35742-35750.

55. Arnoult D, Rismanchi N, Grodet A, Roberts RG, Seeburg DP, Estaquier J et al. Bax/Bak-dependent release of DDP/TIMM8a promotes Drp1-mediated mitochondrial fission and mitoptosis during programmed cell death. Curr Biol 2005; 15 : 2112-2118.

56. Sugioka R, Shimizu S, Tsujimoto Y. Fzo1, a protein involved in mitochondrial fusion inhibits apoptosis. J Biol Chem 2004; 279: 52726-52734.

57. Neuspiel M, Zunino R, Gangaraju S, Rippstein P, McBride HM. Activated Mfn2 signals mitochondrial fusion, interferes with Bax activation and reduces susceptibility to radical induced depolarization. J Biol Chem 2005; 280: 25060-25070.

58. Germain M, Mathai JP, McBride HM, Shore GC. Endoplasmic reticulum BIK initiates DRP1-regulated remodelling of mitochondrial cristae during apoptosis. EMBO J 2005; 24 : 1546-1556

59. Alirol E, James D, Huber D, Marchetto A, Vergani L, Martinou JC et al. The mitochondrial fission protein hFis1 requires the endoplasmic reticulum gateway to induce apoptosis. Mol Biol Cell 2006; 17: 4593-4605.

60. Olichon A, Baricault L, Gas N, Guillou E, Valette A, Belenguer P et al. Loss of OPA1 perturbates the mitochondrial inner membrane structure and integrity, leading to cytochrome c release and apoptosis. J Biol Chem 2003; 278: 7743-7746.
61. Hinshaw JE, Schmid SL. Dynamin self-assembles into rings suggesting a mechanism for coated vesicle budding. Nature 1995; 374: 190-192.

62. Frezza C, Cipolat S, de Brito OM, Micaroni M, Beznoussenko GV, Bartoli D et al. OPA1 controls mitochondrial cristae remodelling independently from mitochondrial fusion. Cell 2006; 126: 177-189.

63. Sesaki H, Southard SM, Hobbs AE, Jensen RE. Cells lacking Pcp1p/Ugo2p, a rhomboidlike protease required for Mgm1p processing, lose mtDNA and mitochondrial structure in a Dnm1p-dependent manner, but remain competent for mitochondrial fusion. Biochem Biophys Res Commun 2003; 308: 276-283

64. Herlan M, Vogel F, Bornhovd C, Neupert W, Reichert AS. Processing of Mgm1 by the rhomboid-type protease Pcp1 is required for maintenance of mitochondrial morphology and of mitochondrial DNA. J Biol Chem 2003; 278: 27781-27788.

65. McQuibban GA, Saurya S, Freeman M. Mitochondrial membrane remodelling regulated by a conserved rhomboid protease. Nature 2003; 423: 537-541.

66. Koonin EV, Makarova KS, Rogozin IB, Davidovic L, Letellier MC, Pellegrini L. The rhomboids: a nearly ubiquitous family of intramembrane serine proteases that probably evolved by multiple ancient horizontal gene transfers. Genome Biol 2003; 4: R19.

67. Urban S. Rhomboid proteins: conserved membrane proteases with diverging biological functions. Genes Dev 2006; 20: 3054-3068.

68. Weihofen A, Martoglio B. Intramembrane-cleaving proteases: controlled liberation of proteins and bioactive peptides. Trends Cell Biol 2003; 13: 71-78

69. Rawson RB, Zelenski NG, Nijhawan D, Ye J, Sakai J, Hasan MT et al. Complementation cloning of S2P, a gene encoding a putative metalloprotease required for intramembrane cleavage of SREBPs. Mol Cell 1997; 1: 45-57.

70. De Strooper B, Saftig P, Craessaerts K, Vanderstichele H, Guhde G, Annaert W et al. Deficiency of presenilin-1 inhibits the normal cleavage of amyloid precursor protein. Nature 1998; 391: 387-390.

71. De Strooper B, Annaert W, Cupers P, Saftig P, Craessaerts K, Mumm JS et al. A presenilin-1-dependent gamma-secretase-like protease mediates release of Notch intracellular domain. Nature 1999; 398: 518-522.

72. Weihofen A, Binns K, Lemberg MK, Ashman K, Martoglio B. Identification of signal peptide peptidase, a presenilin-type aspartic protease. Science 2002; 296: 2215-2218.

73. Urban S, Wolfe MS. Reconstitution of intramembrane proteolysis in vitro reveals that pure rhomboid is sufficient for catalysis and specificity. Proc Natl Acad Sci USA 2005; 102: $1883-1888$.

74. Wang $\mathrm{Y}$, Zhang $\mathrm{Y}, \mathrm{Ha} \mathrm{Y}$. Crystal structure of a rhomboid family intramembrane protease. Nature 2006; 444: 179-180.

75. Bier E, Jan LY, Jan YN. rhomboid, a gene required for dorsoventral axis establishment and peripheral nervous system development in Drosophila melanogaster. Genes Dev 1990; 4: 190-203.

76. Sesaki $H$, Jensen RE. UGO1 encodes an outer membrane protein required for mitochondrial fusion. J Cell Biol 2001; 152: 1123-1134.

77. Sik A, Passer BJ, Koonin EV, Pellegrini L. Self-regulated cleavage of the mitochondrial intramembrane-cleaving protease PARL yields Pbeta, a nuclear-targeted peptide. J Biol Chem 2004; 279: 15323-15329.

78. Jeyaraju D, Xu L, Letellier MC, Bandaru S, Zunino R, Berg EA et al. Phosphorylation and cleavage of a vertebrate-specific domain of the rhomboid protease PARL regulate mitochondrial morphology. Proc Natl Acad Sci USA 2006; 103: 18562-18567.

79. Ishihara N, Fujita Y, Oka T, Mihara K. Regulation of mitochondrial morphology through proteolytic cleavage of OPA1. EMBO J 2006; 25: 2966-2977.

80. Duvezin-Caubet S, Jagasia R, Wagener J, Hofmann S, Trifunovic A, Hansson A et al. Proteolytic processing of OPA1 links mitochondrial dysfunction to alterations in mitochondrial morphology. J Biol Chem 2006; 281: 37972-37979.

81. Olichon A, Emorine LJ, Descoins E, Pelloquin L, Brichese L, Gas N et al. The human dynamin-related protein OPA1 is anchored to the mitochondrial inner membrane facing the inter-membrane space. FEBS Lett 2002; 523: 171-176.

82. Koppen M, Metodiev MD, Casari G, Rugarli El, Langer T. Variable and tissue-specific subunit composition of mitochondrial m-AAA protease complexes linked to hereditary spastic paraplegia. Mol Cell Biol 2007; 27: 758-767.

83. Gakh O, Cavadini P, Isaya G. Mitochondrial processing peptidases. Biochim Biophys Acta 2002; 1592: 63-77.

84. Misaka T, Miyashita T, Kubo Y. Primary structure of a dynamin-related mouse mitochondrial GTPase and its distribution in brain, subcellular localization, and effect on mitochondrial morphology. J Biol Chem 2002; 277: 15834-15842.

85. Harris RA, Hawes JW, Popov KM, Zhao Y, Shimomura Y, Sato J et al. Studies on the regulation of the mitochondrial alpha-ketoacid dehydrogenase complexes and their kinases. Adv Enzyme Regul 1997; 37: 271-293.

86. Roche TE, Baker JC, Yan X, Hiromasa Y, Gong X, Peng T et al. Distinct regulatory properties of pyruvate dehydrogenase kinase and phosphatase isoforms. Prog Nucleic Acid Res Mol Biol 2001; 70: 33-75.

87. Horbinski $C$, Chu CT. Kinase signaling cascades in the mitochondrion: a matter of life or death. Free Radic Biol Med 2005; 38: 2-11.

88. Valente EM, Abou-Sleiman PM, Caputo V, Muqit MM, Harvey K, Gispert S et al. Hereditary early-onset Parkinson's disease caused by mutations in PINK1. Science 2004; 304: 1158-1160. 
89. Pagliarini DJ, Wiley SE, Kimple ME, Dixon JR, Kelly P, Worby CA et al. Involvement of a mitochondrial phosphatase in the regulation of ATP production and insulin secretion in pancreatic beta cells. Mol Cell 2005; 19: 197-207.

90. Butow RA, Avadhani NG. Mitochondrial signaling: the retrograde response. Mol Cell 2004; 14: $1-15$.

91. Epstein CB, Waddle JA, Hale Wt, Dave V, Thornton J, Macatee TL et al. Genome-wide responses to mitochondrial dysfunction. Mol Biol Cell 2001; 12: 297-308.

92. Traven $A$, Wong JM, Xu D, Sopta M, Ingles CJ. Interorganellar communication. Altered nuclear gene expression profiles in a yeast mitochondrial DNA mutant. J Biol Chem 2001; 276: 4020-4027.
93. Loveland $\mathrm{B}$, Wang $\mathrm{CR}$, Yonekawa $\mathrm{H}$, Hermel $\mathrm{E}$, Lindahl KF. Maternally transmitted histocompatibility antigen of mice: a hydrophobic peptide of a mitochondrially encoded protein. Cell 1990; 60: 971-980.

94. Augustin S, Nolden M, Muller S, Hardt O, Arnold I, Langer T. Characterization of peptides released from mitochondria: evidence for constant proteolysis and peptide efflux. $J$ Biol Chem 2005; 280: 2691-2699.

95. Arnold I, Wagner-Ecker M, Ansorge W, Langer T. Evidence for a novel mitochondriato-nucleus signalling pathway in respiring cells lacking i-AAA protease and the $A B C$ transporter Mdl1. Gene 2006; 367: 74-88. 